\title{
Quantum evolution according to real clocks
}

\author{
Iñigo L. Egusquiza, ${ }^{1}$ Luis J. Garay, ${ }^{2}$ and José M. Raya ${ }^{3}$ \\ ${ }^{1}$ Fisika Teorikoaren Saila, Euskal Herriko Unibertsitatea, 644 Posta Kutxa, 48080 Bilbao, Spain \\ ${ }^{2}$ Instituto de Matemáticas y Física Fundamental, CSIC, C/ Serrano 121, 28006 Madrid, Spain \\ ${ }^{3}$ Instituto de Astrofísica de Andalucía, CSIC, Camino Bajo de Huétor, 18080 Granada, Spain
}

(Received 3 November 1998)

\begin{abstract}
We characterize good clocks, which are naturally subject to fluctuations, in statistical terms. We also obtain the master equation that governs the evolution of quantum systems according to these clocks and find its general solution. This master equation is diffusive and produces loss of coherence. Moreover, real clocks can be described in terms of effective interactions that are nonlocal in time. Alternatively, they can be modeled by an effective thermal bath coupled to the system. [S1050-2947(99)04905-7]

PACS number(s): 03.65.Bz, 05.40.-a, 42.50.Lc
\end{abstract}

\section{INTRODUCTION}

In quantum mechanics, spatial positions are described by quantum observables. However, the situation with respect to the time parameter is rather more involved, as has been known since Pauli pointed out that there is no self-adjoint operator canonically conjugate to the total energy, if the spectrum of the latter is bounded from below [1]. Some attempts have been made to circumvent this problem by making use of the concept of time of arrival [2]. Recent developments, however, have mapped the problem of constructing a good time of arrival operator into the problem of constructing a good-time operator [3], thus showing that the special status of time is deeply embedded into the structure of quantum mechanics. We are then bound to use real physical clocks and rely on their readouts when measuring the evolution of a quantum system.

Any real clock is inevitably subject to quantum fluctuations, which introduce uncertainties in the equations of motion. For instance, it has been shown that the finite mass and size of the clock impose limitations in the measurement of space-time distances in the framework of general relativity [4]. Some considerations have also been made on the role of quantum clocks in the context of quantum cosmology $[5,6]$. Simple models for quantum clocks have been proposed, and the quantum evolution of a system according to a quantum clock suitably coupled with it has also been studied [4-9]. The general conclusion is that the system becomes more and more perturbed as the resolution of the clock is improved. Even more, quantum gravity may well imply the existence of an absolute limit, the Planck scale, to the accuracy of spacetime-distance measurements and, in particular, to clock synchronization (for a review, see, e.g., Ref. [10]), with possible effects in the low-energy regime [11].

It follows that any quantum clock that we could possibly build would lead to uncertainties and errors. These quantum errors, however, are not the only source of randomness in the measure of time. Real clocks are also subject to classical imperfections, small errors, that can only be dealt with statistically. For instance, an unavoidable classical source of stochasticity is temperature, which will introduce thermal fluctuations in the behavior of real clocks. Although this is not necessarily the most important source of errors in mod- ern day atomic clocks, it is nonetheless always present to some extent. In other words, the third law of thermodynamics forbids the existence of ideal clocks.

We will study, within the context of the standard quantum theory, the evolution of an arbitrary system according to a real clock. Unlike other works [4-9], we are not concerned about the quantum-dynamical behavior of the clock but only with the readings of time that it provides. As stated before, these readings will undergo errors, which will be described by a stochastic process. It is in this sense that we regard these clocks as classical, although this does not preclude a possible quantum-mechanical origin for the stochastic fluctuations. Notice also that we will mostly consider clocks which are decoupled from the system under study. In what follows we shall not delve further into the source of stochasticity, but assume a phenomenological description of it.

Let us be more specific about the meaning of this randomness in the readings of the clock. Imagine a large ensemble of identical systems, prepare one of them in a given initial state at initial clock time $t=0$, and then measure the state of that system at clock time $t$. If we repeat this procedure for all the systems in the ensemble, the result will be a probability distribution for the possible outcomes, its dispersion partially being a consequence of the lack of knowledge of the precise ideal time that has elapsed. Therefore, the evolution according to the readouts of the real clock is nonunitary. In other words, the use of real clocks induces loss of coherence in most physical quantum states, as we will explicitly show.

To attain this objective we will start by analyzing in some detail the characteristics that should be expected from a good real clock. This is expounded in the next section. The implications of the randomness in the readings of a real clock for the quantum evolution of a system is explored in Sec. III. In Sec. IV, we discuss the evolution according to real clocks from an effective point of view in terms of nonlocal interactions in time and also in terms of a thermal bath coupled to the system under study. Decoherence owing to clock errors is investigated in Sec. V. Finally, we summarize and conclude in the last section with some general remarks.

\section{GOOD CLOCKS}

Let us consider the phase space of a classical physical system, divided in a set of ordered cells, such that the ex- 
pected evolution of the system moves the state from one cell $k$ to the next $k+1$ in a time $\varepsilon$ approximately. An observer keeps tally of which is the cell of largest $k$ that has been visited, and thus the passage of time is recorded. Let $s_{k}$ denote the ideal time at which the readout of the clock is $k \varepsilon$, i.e., $s_{k}$ is the ideal time of first arrival of the system at the $k$ th cell. For an ideal clock, the time $s_{k}$ and the clock time $k \varepsilon$ would coincide. However, for a real clock, these two quantities will differ by an error $\Delta_{k}=s_{k}-k \varepsilon$, where it should be noticed that the index $k$ pertains to the readout $k \varepsilon$ of the clock, i.e., to the $k$ th tick, and not to a preset ideal time. Given any real discrete clock, its characteristics will be encoded in the probability distribution for the stochastic sequence [12] of clock errors, $P\left(\left\{\Delta_{k}\right\}\right)$, which must satisfy appropriate conditions, so that it describes a good clock.

A first property is that Galilean causality should be preserved, i.e., that causally related events should always be properly ordered in clock time as well, which implies that $s_{k+1}>s_{k}$ for every $k$. In terms of the discrete derivative $\alpha_{k}$ $=\left(\Delta_{k+1}-\Delta_{k}\right) / \varepsilon$ of the stochastic sequence $\left\{\Delta_{k}\right\}$, we can state this condition as requiring that, for any realization of the stochastic sequence, $\alpha_{k}>-1$.

A second condition that we would require good clocks to fulfill is that the expectation value of relative errors, determined by the stochastic sequence $\left\{\alpha_{k}\right\}$, be zero, i.e., $\left\langle\alpha_{k}\right\rangle$ $=0$ for all $k$. If this were not the case, the clock would either systematically go fast or slow down, and a redefinition through this systematic drift would provide us with a wellcentered clock. Consequently, the expectation value for the absolute errors $\left\{\Delta_{k}\right\}$ will be constant. Furthermore, since $k$ $=0$ will be the time at which the systems whose evolution we are studying are prepared, $\Delta_{0}$ will not be stochastic and, without loss of generality, will be set to zero by a simple translation of the origin of time, so that $\left\langle\Delta_{k}\right\rangle=0$.

Another expectation that we would have for a good clock is that it should always behave in the same way (in a statistical sense). The difference between the ideal time for the $(k+1)$ th tick and the ideal time for the $k$ th tick must be always the same, statistically speaking, even if the actual errors $\Delta_{k+1}$ and $\Delta_{k}$ are large by some statistical fluctuation. Therefore, we can say that the clock behaves consistently in time as a good one if those relative errors $\left\{\alpha_{k}\right\}$ are statistically stationary, i.e., the probability distribution $\mathcal{P}\left(\left\{\alpha_{k}\right\}\right)$ for the sequence of relative errors $\left\{\alpha_{k}\right\}$ [which can be obtained from $P\left(\left\{\Delta_{k}\right\}\right)$, and vice versa $]$ must not be affected by global shifts $k \rightarrow k+k_{0}$ of the readout of the clock. Note that the stochastic process $\Delta_{k}$ need not be stationary, despite the stationarity of the process $\alpha_{k}$.

It is also intuitively obvious that the one-point probability distribution function for the variables $\alpha_{k}$ should be highly concentrated around the zero mean, if the clock is to behave nicely. Even more, it is to be expected for clocks with small errors that all the higher-order cumulants be much smaller than the correlation, which, in turn, should also be bounded by a small number, i.e.,

$$
\left\langle\alpha_{k}\right\rangle=0, \quad\left\langle\alpha_{k} \alpha_{k-j}\right\rangle \equiv c_{j} \leqslant c_{0} \ll 1,
$$

where $c_{j}=c_{-j}$. The correlation for the sequence of absolute errors $\left\{\Delta_{k}\right\}$ can then be easily obtained and has the form $\left\langle\Delta_{k} \Delta_{l}\right\rangle=\varepsilon^{2} \sum_{i=1}^{k} \Sigma_{j=1}^{l} c_{i-j}$.
The correlation time $\vartheta$ for the stochastic sequence $\left\{\alpha_{k}\right\}$ is given by $\vartheta=\varepsilon \sum_{j=-\infty}^{+\infty} c_{j} /\left(2 c_{0}\right)$. We will introduce a new parameter $\kappa$ with dimensions of time, defined as $\kappa^{2}$ $=c_{0} \vartheta^{2}$. This comes about because, when the errors of the clock have a thermal origin, $\kappa^{2}$ is proportional to the temperature, and independent of $\vartheta$. In general, the good-clock conditions imply $\kappa \ll \vartheta$. As we shall see, $\vartheta$ cannot be arbitrarily large, and, therefore, the ideal clock limit is given by $\kappa \rightarrow 0$.

Until now we have discussed general properties that a good clock must fulfill, regardless of the physical system under study. In addition to these properties, a good clock must have enough precision in order to measure the evolution of the specific system, which imposes further restrictions on the clock. On the one hand, the characteristic evolution time $\zeta$ of the system must be much larger than the correlation time $\vartheta$ of the clock. On the other hand, the leading term in the asymptotic expansion of the variance $\left\langle\Delta_{k}^{2}\right\rangle$ for large $k$ is of the form $\kappa^{2}(k \varepsilon / \vartheta)$ which means that, after a certain period of time, the absolute errors can be too large. The maximum admissible standard deviation in $\Delta_{k}$ must be at most of the same order as $\zeta$. Then the period of applicability of the clock to the system under study, i.e., the period of clock time during which the errors of the clock are smaller than the characteristic evolution time of the system is approximately equal to $\zeta^{2} \vartheta / \kappa^{2}$. For a good clock, $\kappa \ll \vartheta \ll \zeta$, as we have seen, so that the period of applicability is much larger than the characteristic evolution time $\zeta$.

Even though, so far, we have only spoken of good discrete clocks, by analogy, we will consider continuous stochastic processes $\alpha(t)$, with corresponding probability functionals $\mathcal{P}[\alpha(t)]$. The conditions previously stated for the discrete sequence $\left\{\alpha_{k}\right\}$ admit a straight-forward generalization to the continuous case. In what follows, we shall use the formulation in the continuum.

\section{MASTER EQUATION}

We shall now obtain the evolution equation for the density matrix of an arbitrary quantum system in terms of the clock time $t$. Let $\rho_{S}(s)$ be the density matrix for a quantum system whose unitary evolution in the ideal Schrödinger time $s=t+\Delta(t)$ is provided by the time-independent Hamiltonian $H$.

For any given realization of the stochastic process that characterizes a good clock, and using the chain rule, we can write the von Neumann evolution equation in terms of the clock time $t$ as

$$
\partial_{t} \rho_{S}(t+\Delta(t))=-i(1+\alpha(t))\left[H, \rho_{S}(t+\Delta(t))\right],
$$

where $\hbar$ has been set to 1 .

Let us now transform to the interaction picture in which the density matrix has the form

$$
\rho_{S}^{I}(t+\Delta(t))=e^{i H t} \rho_{S}(t+\Delta(t)) e^{-i H t} .
$$

Notice that the interaction term $\alpha(t) H$ has the same form in both pictures because it is proportional to the free Hamiltonian $H$. 
Integrating the resulting equation between 0 and $t$, and reintroducing the result for $\rho_{S}^{I}$, we obtain the following integro-differential equation:

$$
\begin{aligned}
\partial_{t} \rho_{S}^{I}(t+\Delta(t))= & -i \alpha(t)\left[H, \rho_{S}^{I}(0)\right] \\
& -\int_{0}^{t} d t^{\prime} \alpha(t) \alpha\left(t^{\prime}\right)\left[H,\left[H, \rho_{S}^{I}\left(t^{\prime}+\Delta\left(t^{\prime}\right)\right)\right]\right] .
\end{aligned}
$$

In order to find the evolution equation in the time $t$, we have to average this equation over all possible realizations $\alpha(t)$ of the stochastic process with the functional weight $\mathcal{P}[\alpha(t)]$. The average of the density matrix $\rho_{S}^{I}(t+\Delta(t))$ will be denoted by $\rho^{I}(t)$ and can be regarded as the density matrix of the system at clock time $t$.

At the real time $t=0$, we impose the initial condition $\rho^{I}(0)=\rho_{S}^{I}(0)=\rho_{0}^{I}$. Additionally, for a good clock, $\langle\alpha(t)\rangle$ $=0$, as already discussed, and, as a consequence, the average of the linear term in $\alpha(t)$ vanishes. Furthermore, the clock time derivative $\partial_{t}$ and the average over $\alpha\left(t^{\prime}\right)$ commute because $\mathcal{P}\left[\alpha\left(t^{\prime}\right)\right]$ is stationary. Finally, the density matrix $\rho_{S}^{I}\left(t^{\prime}+\Delta\left(t^{\prime}\right)\right)$ can be expanded in powers of $\Delta\left(t^{\prime}\right)$. Then the average of the integro-differential equation for the density matrix $\rho^{I}$ yields

$$
\dot{\rho}^{I}(t)=-\int_{0}^{t} d \tau c(\tau)\left[H,\left[H, \rho^{I}(t-\tau)\right]\right]+O\left(\left\langle\alpha^{3}\right\rangle\right),
$$

where the overdot denotes derivative with respect to the clock time $t$. We have also performed a change of the integration variable from $t^{\prime}$ to $\tau=t-t^{\prime}$ and have introduced the correlation function $c(\tau)$ for the stochastic process $\alpha(t)$.

For a good clock, the higher-order terms in $\alpha$ can be seen to be much smaller than the $c(\tau)$ term by a factor $(\kappa / \zeta)^{2}$ $\ll 1$, provided that the system evolves for a time smaller than the period of applicability of the clock. Since $\zeta \gg \vartheta$, the system does not evolve significantly within a correlation time, and we can substitute $\rho^{I}(t-\tau)$ by $\rho^{I}(t)$. This is the so-called Markov approximation. The process $\Delta(t)$ will not be Markovian in general and there is no reason for requiring that the process $\alpha(t)$ has this property either. However, and even though the Markov approximation refers to the system and not to the clock itself, it renders the possible non-Markovian character of the clock irrelevant. Furthermore, for evolution times $t$ much larger than the correlation time $\vartheta$, we can take the upper integration limit to infinity.

The resulting master equation, once we go back to the Schrödinger picture, can be written as

$$
\dot{\rho}(t)=-i[H, \rho(t)]-\left(\kappa^{2} / \vartheta\right)[H,[H, \rho(t)]] .
$$

Notice that the accuracy of the clock appears in the master equation through the parameters $\kappa$ and $\vartheta$ and that, in the ideal clock limit, $\kappa \rightarrow 0$, the unitary von Neumann equation is recovered. We should also point out that this master equation is not a truncation of the BBGKY hierarchy [13], and that irreversibility appears because the errors of the clock cannot be eliminated once we have started using it.

Under the good-clock conditions, $\kappa \ll \vartheta$, we can approximate $\mathcal{P}[\alpha(t)]$ by a stationary Gaussian probability functional with zero mean and correlation given by the correlation $c(t)$ of $\mathcal{P}[\alpha(t)]$. Although this Gaussian approximation assigns a nonvanishing probability to $\alpha(t)<-1$, this probability will be negligibly small since, for good clocks, $c(t) \ll 1$. Thus the Gaussian approximation to good clocks fulfills the Galilean causality condition for all practical purposes.

In the Gaussian approximation, there is essentially only one good clock for which $\alpha(t)$ is Markovian, the OrnsteinUhlenbeck process [12]. In this case, the correlation function for $\alpha(t)$ in the stationary regime is $c(\tau)=(\kappa / \vartheta)^{2} e^{-|\tau| / \vartheta}$. Since the possible non-Markovian character of the clock does not influence the time evolution of the system (provided that the condition $\zeta \ll \vartheta$ is satisfied, as happens for good clocks), the Ornstein-Uhlenbeck clock is generic in what concerns the evolution of quantum systems according to real clocks.

\section{EFFECTIVE DESCRIPTIONS}

The master equation corresponds to the evolution of a system with a free Hamiltonian $H$ coupled with a classical noise source $\alpha(t)$, with a probability functional distribution $\mathcal{P}[\alpha(t)]$, via the interaction Hamiltonian $\alpha(t) H$. The path integrals for this system then follow the pattern [14],

$$
\int \mathcal{D} \alpha \mathcal{P}\left[\alpha\left(t^{\prime}\right)\right] \int \mathcal{D} q \mathcal{D} p e^{i\left[\int d t(p \dot{q}-H)-\int d t \alpha(t) H(t)\right]} .
$$

In the good-clock approximation, only the two-point correlation function $c(\tau)$ is relevant, so that we can write the probability functional as a Gaussian distribution. The integration over $\alpha(t)$ is then easily performed to yield

$$
\int \mathcal{D} q \mathcal{D} p e^{i \int d t(p \dot{q}-H)-(1 / 2) \int d t d t^{\prime} c\left(t-t^{\prime}\right) H(t) H\left(t^{\prime}\right)} .
$$

Therefore, we see that the effect of using real clocks for studying the evolution of a quantum system is the appearance of an effective interaction term in the action integral, which is bilocal in time. This can be understood as the first term in a multilocal expansion, which corresponds to the weak-field expansion of the probability functional around the Gaussian term.

This nonlocality in time admits a simple interpretation: correlations between relative errors at different instants of clock time can be understood as correlations between clocktime flows at those clock instants. The clock-time flow of the system is governed by the Hamiltonian and, therefore, the correlation of relative errors induces an effective interaction term, generically multilocal, that relates the Hamiltonians at different clock instants.

The expression for the influence functional is, in the weak-noise limit, completely analogous to the expressions above. Since the noise source is classical, we see that there is no dissipative term there, nor in the master equation [14]. Moreover, as the interaction term is proportional to $H$, there is no response of the system to the outside noise, which means that the associated impedance is infinite $[15,16]$.

From a different point of view, the clock can be effectively modeled by a thermal bath, with a temperature $T_{b}$ to be determined, coupled to the system. Let $H+H_{\text {int }}+H_{b}$ be the total Hamiltonian, where $H$ is the free Hamiltonian of the system and $H_{b}$ is the Hamiltonian of a bath that will be 
represented by a collection of harmonic oscillators [16]. The interaction Hamiltonian will be of the form $H_{\text {int }}=\xi H$, where the noise operator $\xi$ is given by

$$
\xi(t)=\frac{i}{\sqrt{2 \pi}} \int_{0}^{\infty} d \omega \chi(\omega)\left[a^{\dagger}(\omega) e^{i \omega t}-a(\omega) e^{-i \omega t}\right] .
$$

In this expression, $a$ and $a^{\dagger}$ are, respectively, the annihilation and creation operators associated with the bath, and $\chi(\omega)$ is a real function, to be determined, that represents the coupling between the system and the bath for each frequency $\omega$.

Identifying, in the classical noise limit, the classical correlation function of the bath with $c(\tau)$, the suitable coupling between the system and the bath is given by the spectral density of fluctuations of the clock:

$$
k_{B} T_{b} \chi(\omega)^{2}=\int_{0}^{\infty} d \tau c(\tau) \cos (\omega \tau)
$$

where $k_{B}$ is Boltzmann's constant. With this choice, the master equation for evolution according to real clocks is identical to the master equation for the system obtained by tracing over the effective bath.

To go beyond the classical noise limit requires the introduction of the usual quadratic dissipation term in the influence functional [14]. However, the peculiar coupling to the energy $\xi H$, which is quite different from the usual coupling to the position or the momentum of the system, implies that this term does not produce dissipation in the equations of motion: the fluctuation-dissipation theorem, which reflects the microscopic structure of the bath, is thus fulfilled, but there is no dissipation.

\section{DECOHERENCE}

The master equation contains a diffusion term and will therefore lead to a loss of coherence [17]. However, this loss depends on the initial state. In other words, there exists a pointer basis [18], so that any density matrix which is diagonal in this specific basis will not be affected by the diffusion term, while any other will approach a diagonal density matrix. The stochastic perturbation $\alpha(t) H$ is obviously diagonal in the basis of eigenstates $\{|n\rangle\}$ of the Hamiltonian, which is therefore the pointer basis: the interaction term cannot induce any transition between different energy levels $\omega_{n}$.

The components of the density matrix in this basis are $\rho_{n m}=\langle n|\rho| m\rangle$. The master equation can be solved exactly, its general solution being

$$
\rho_{n m}(t)=\rho_{n m}(0) e^{-i \omega_{n m} t} e^{-\left(\omega_{n m}\right)^{2} \kappa^{2} t / \vartheta},
$$

where $\omega_{n m}=\omega_{n}-\omega_{m}$. The smallest energy difference $\omega$ provides the inverse of the characteristic time for the evolution of the system, $\zeta=1 / \omega$. The smallest decay constant is $\omega^{2} \kappa^{2} / \vartheta$, equal to the inverse of the period of applicability of the clock. By the end of this period, the density matrix will have been reduced to the diagonal terms and a much diminished remnant of those off-diagonal terms with slow evolution. In any case, the von Neumann entropy grows if the density matrix is not initially diagonal in the energy basis.
The effect of decoherence due to errors of real clocks does not only turn up in the quantum context. Consider, for instance, a classical particle with a definite energy $E$ moving under a time-independent Hamiltonian $H$. Because of the errors of the clock, we cannot be positive about the location of the particle in its trajectory on phase space at our clock time $t$. Therefore, we have an increasing spread in the coordinate and conjugate momentum over the trajectory. For a generic system, this effect is codified in the classical master equation,

$$
\dot{\varrho}=\{H, \varrho\}+\left(\kappa^{2} / \vartheta\right)\{H,\{H, \varrho\}\},
$$

where $\varrho(t)$ is the probability distribution on phase space in clock time. This classical master equation can be derived in a manner completely analogous to the quantum one.

For simplicity, let us study the particular example of a one-dimensional Hamiltonian motion with closed orbits, with $H=\omega J, \varphi$ being the angle variable with period $2 \pi$ conjugate to the action variable $J$, and $\omega$ a constant frequency characteristic of the system. The classical master equation for the probability density $\varrho(\varphi, J ; t)$ reads

$$
\partial_{t} \varrho=\omega \partial_{\varphi} \varrho+\left(\omega^{2} \kappa^{2} / \vartheta\right) \partial_{\varphi}^{2} \varrho .
$$

This diffusion equation can be exactly solved by separation of variables. The slowest decaying mode has, as before, a decay constant $\omega^{2} \kappa^{2} / \vartheta$.

In the case of one particle that is released with energy $E$ and initial angle $\varphi_{0}$, the probability distribution spreads out over the corresponding connected component of the energy shell, and tends to $\delta(J-E / \omega) / 2 \pi$ as clock time grows. As we can see, the information about the $\varphi$ variable is washed out by the errors in our clock, which is precisely the information that is not available in the quantum case: if $J$ is completely known for a given quantum state, the indeterminacy in its conjugate variable will be infinite, the situation towards which the classical decoherence process tends.

Finally, it should be observed that the mechanism of decoherence is neither tracing over degrees of freedom, nor coarse graining, nor dephasing $[17,19]$. Even though there is no integration over time introduced here by fiat, as happens in dephasing in quantum mechanics, the spread in time due to the errors of the clock has a similar effect, and produces decoherence.

\section{CONCLUSIONS}

In our study of the evolution of quantum systems according to real clocks, which are necessarily subject to errors, we have first established a stochastic characterization of good real clocks. Using this description, we have derived a master equation for the quantum evolution in real-clock time and we have also found its general solution in the basis of energy eigenstates. The stochastic features of good real clocks and their effects on the quantum evolution can be equivalently described by means of interactions which are nonlocal in time. They can also be effectively modeled by a quantum thermal bath. The master equation exhibits a diffusion term, which is responsible for the loss of coherence of most initial states. Finally, we have analyzed the evolution of classical 
systems according to real clocks and reached analogous conclusions.

The third law of thermodynamics and the quantum fluctuations prevent real clocks from being perfectly accurate. This suggests that, strictly speaking, the Schrödinger unitary evolution equation is just an excellent approximation valid for sufficiently short periods of time and that should be substituted, along the lines proposed in this paper, by a diffusive master equation in more general situations. This adds a random aspect to the evolution of quantum systems. Indeed, coherence is progressively lost until we reach the period of applicability of the clock and, after that time, unpredictability sets in, as we have seen. Even perfectly isolated systems will suffer loss of coherence because of the fluctuations of the real clock and will appear as effectively coupled to a reservoir.

\section{ACKNOWLEDGMENTS}

We thank C. Barceló, C. Cabrillo, P.F. González-Díaz, G.A. Mena Marugán, and M.A. Valle Basagoiti for discussions. J.M.R. is also grateful to J.M. Quintana. We had support from the University of the Basque Country (Project No. UPV 063.310-EB225/95) from Junta de Andalucía, and from DGICYT (Spain) under Project Nos. PB94-0107 and PB930139.
[1] W. Pauli, Die Allgemeinen Prinzipien der Wallenmechanik. Handbuch der Physik, edited by S. Flügge (Springer, Berlin, 1958), Vol. V/1, p. 60; General Principles of Quantum Mechanics (Springer-Verlag, Berlin, 1980), p. 63 (English version).

[2] J. G. Muga, R. Sala, and J. P. Palao, Superlattices Microstruct. 23, 833 (1998).

[3] J. G. Muga, C. R. Leavens, and J. P. Palao, Phys. Rev. A 58, 4336 (1998); J. Oppenheim, B. Reznik, and W. G. Unruh, Time-of-Arrival States, Report No. quant-ph/9807043.

[4] E. Wigner, Rev. Mod. Phys. 29, 255 (1957); H. Salecker and E. Wigner, Phys. Rev. 109, 571 (1958).

[5] J. B. Hartle, Phys. Rev. D 38, 2985 (1988).

[6] W. G. Unruh and R. M. Wald, Phys. Rev. D 40, 2598 (1989).

[7] A. Peres, Am. J. Phys. 48, 552 (1980).

[8] D. N. Page and W. K. Wootters, Phys. Rev. D 27, 2885 (1983).

[9] Y. Aharanov et al., Phys. Rev. A 57, 4130 (1998).

[10] L. J. Garay, Int. J. Mod. Phys. A 10, 145 (1995).

[11] S. W. Hawking, Commun. Math. Phys. 87, 395 (1982); J. Ellis et al., Nucl. Phys. B 241, 381 (1984); L. J. Garay, Phys. Rev. Lett. 80, 2508 (1998); Phys. Rev. D 58, 124015 (1998);
G. Amelino-Camelia et al., Nature (London) 393, 763 (1998).

[12] N. G. Van Kampen, Stochastic Processes in Physics and Chemistry (North-Holland, Amsterdam, 1981); C. W. Gardiner, Handbook of Stochastic Methods for Physics, Chemistry, and the Natural Sciences (Springer-Verlag, Berlin, 1985).

[13] K. Huang, Statistical Mechanics (Wiley, New York, 1987).

[14] R. P. Feynman and F. Vernon, Ann. Phys. (N.Y.) 24, 118 (1963); R. P. Feynman and A. Hibbs, Quantum Mechanics and Path Integrals (McGraw-Hill, New York, 1965).

[15] H. B. Callen and T. A. Welton, Phys. Rev. 83, 34 (1951).

[16] C. W. Gardiner, Quantum Noise (Springer-Verlag, Berlin, 1991); L. Mandel and E. Wolf, Optical Coherence and Quantum Optics (Cambridge University Press, Cambridge, 1995).

[17] D. Giulini et al., Decoherence and the Appearance of the Classical World in Quantum Theory (Springer-Verlag, Berlin, 1996).

[18] W. H. Zurek, Phys. Rev. D 24, 1516 (1981); 26, 1862 (1982); in The Physical Origins of Time Asymmetry, edited by J. J. Halliwell, J. Pérez-Mercader, and W. H. Zurek (Cambridge University Press, Cambridge, 1994).

[19] F. Cooper et al., Phys. Rev. D 55, 6471 (1997). 